$\mathrm{J}$ o u r n a l of

Mathematics

and Applications

JMA No 42, pp 95-107 (2019)

\title{
Nonincreasing Solutions for Quadratic Integral Equations of Convolution Type
}

\author{
W.G. El-Sayed and A.A.H. Abd El-Mowla
}

ABSTRACT: We study a nonlinear quadratic integral equation of Convolution type in the Banach space of real functions defined and continuous on a bounded and closed interval. By using a suitable measure of noncompactness, we show that the integral equation has monotonic solutions.

AMS Subject Classification: 45G10, 47H30, 47N20.

Keywords and Phrases: Quadratic integral equation; Measure of noncompactness; Fixed-point theorem; Monotonic solutions.

\section{Introduction and preliminaries}

There are many results in nonlinear functional analysis which contain conditions with the measure of noncompactness. Integral equations are one of the most useful mathematical tools in both pure and applied analysis. This is particulary true of problems in mechanical vibrations and the related fields of engineering and mathematical physics. The theory of integral equations is rapidly developing with the help of several tools of functional analysis, topology and fixed point theory. For details, we refer to ([1]-[23]) and the references therein.

The goal of this paper is to study the solvability of the following nonlinear quadratic integral equation

$$
x(t)=g(t)+(T x)(t) \int_{0}^{t} k(t, s) f(s, x(\varphi(s))) d s, t \in[0, M],
$$

in the Banach space of real functions being defined and continuous on a bounded and closed interval. The main tool used to study the existence solutions of that equation in the class of monotonic functions is a special measure of noncompactness. 
Now, we collect some facts, basic concepts and sketch some useful theorems which will be needed further on. Let $(E, \|$. $\|)$ be an infinite dimensional Banach space with zero element $\theta$. Denote by $B(x, r)$ the closed ball in $E$ centered at $x$ with radius $r$. The symbol $B_{r}$ stands for the ball $B(\theta, r)$. If $X$ is a nonempty subset of $E$, then $\bar{X}$ and $\operatorname{Conv} X$ denote the closure and the convex closure of $X$, respectively. Moreover, the symbol $m_{E}$ denotes the family of all nonempty and bounded subsets of $E$ while $n_{E}$ stands for its subfamily consisting of all relatively compact sets.

We will accept the following definition of the concept of a measure of noncompactness $[4]$.

Definition 1. A mapping $\mu: m_{E} \rightarrow[0, \infty)$ is said to be a measure of noncompactness in $E$ if the following conditions are satisfied:

1. the family $\operatorname{ker} \mu=\left\{X \in m_{E}: \mu(X)=0\right\}$ is nonempty and ker $\mu \subset n_{E}$.

2. $X \subset Y \Rightarrow \mu(X) \leq \mu(Y)$.

3. $\mu(X)=\mu(\bar{X})=\mu(\operatorname{Conv} X)$.

4. $\mu(\lambda X+(1-\lambda) Y) \leq \lambda \mu(X)+(1-\lambda) \mu(Y)$ for $\lambda \in[0,1]$.

5. If $\left(X_{n}\right), n \in N$ is sequence of closed sets from $m_{E}$ such that $X_{n+1} \subset X_{n}$ and if $\lim _{n \rightarrow \infty} \mu\left(X_{n}\right)=0$, then the set $X_{\infty}=\bigcap_{n=1}^{\infty} X_{n}$ is nonempty.

The family $\operatorname{ker}(\mu)$ describe in 1 is referred to as the kernel of the measure of noncompactness $\mu$.

A measure $\mu$ is called sublinear if it satisfies the following two conditions:

6. $\mu(\lambda X)=|\lambda| \mu(X)$, for $\lambda \in R$

7. $\mu(X+Y) \leq \mu(X)+\mu(Y)$.

Moreover, a measure $\mu$ is called a measure with maximum property if

8. $\mu(X \bigcup Y)=\max [\mu(X), \mu(Y)]$.

Other facts concerning measures of noncompactness and their properties may be found in [4].

Definition 2. \{Darbo condition\} Let $M$ be a nonempty subset of a Banach space $E$ and the operator $F: M \rightarrow E$ is continuous and transforms bounded sets onto bounded ones, then $F$ satisfies the Darbo condition with constant $k \geq 0$ with respect to a measure of noncompactness $\mu$ if for any bounded subset $X$ of $M$ we have

$$
\mu(F X) \leq k \mu(X) .
$$

If $F$ satisfies the Darbo condition with $k<1$, then it is called a contraction with respect to $\mu$. Next, we need the following fixed point theorem ([4], [16]). 
Theorem 1. Let $Q$ be nonempty bounded closed convex subset of the space $E$ and let $F: Q \rightarrow Q$ be continuous and such that $\mu(F X) \leq k \mu(X)$ for any nonempty subset $X$ of $Q$, where $k$ is a constant, $k \in[0,1)$. Then $F$ has a fixed point in the set $Q$.

Remark 1. Under the assumptions of the above theorem, it can be shown that the set $f i x F$ of fixed points of $F$ belonging to $Q$ is a member of the family ker $\mu$ [4]. This fact permits us to characterize solutions of considered operator equations.

We will work in the classical Banach space $C[0, M]$ consisting of all real functions defined and continuous on the interval $[0, M]$. For convenience, we write $I=[0, M]$ and $C(I)=C[0, M]$. The space $C(I)$ is furnished by the standard norm

$$
\|x\|=\max \{|x(t)|: t \in I\} .
$$

Now, we will display the definition of a measure of noncompactness in $C(I)$. That measure was introduced and studied in [5].

To do this, let us fix a nonempty and bounded subset $X$ of $C(I)$. For $x \in X$ and $\epsilon \geq 0$ denoted by $\omega(x, \epsilon)$, the modulus of continuity of the function $x$, i.e.,

$$
\omega(x, \epsilon)=\sup \{|x(t)-x(s)|: t, s \in I,|t-s| \leq \epsilon\} .
$$

Further, let us put

$$
\begin{gathered}
\omega(X, \epsilon)=\sup \{\omega(x, \epsilon): x \in X\}, \\
\omega_{0}(X)=\lim _{\epsilon \rightarrow 0} \omega(X, \epsilon) .
\end{gathered}
$$

Now, let us define the following quantities:

$$
\begin{gathered}
d(x)=\sup \{|x(s)-x(t)|-[x(s)-x(t)]: t, s \in I, t \leq s\} \\
i(x)=\sup \{|x(t)-x(s)|-[x(t)-x(s)]: t, s \in I, t \leq s\}, \\
d(X)=\sup \{d(x): x \in X\}, \\
i(X)=\sup \{i(x): x \in X\} .
\end{gathered}
$$

Observe that $d(X)=0$ if and only if all functions belonging to $X$ are nondecreasing on $I$. In a similar way, we can characterize the set $X$ with $i(X)=0$.

Finally, we define the function $\mu$ on the family $m_{C(I)}$ by putting

$$
\mu(X)=\omega_{0}(X)+d(X)
$$

It can be shown [5] that the function $\mu$ is a measure of noncompactness in the space $C(I)$. The kernel ker $\mu$ of this measure contains nonempty and bounded sets $X$ such that functions from $X$ are equicontinuous and nondecreasing on the interval $I$. 
Remark 2. By properties of the kernel ker $\mu$ of the measure of noncompactness $\mu$ together with Remark 1 allow us to characterize solutions of the nonlinear integral equation considered in the next section.

Remark 3. Observe that, in a similar way, we can define the measure of noncompactness associated with the set quantity $i(X)$ define above.

\section{Main result}

In this section, we will study the nonlinear quadratic integral equation of Volterra type having the form

$$
x(t)=g(t)+(T x)(t) \int_{0}^{t} k(t, s) f(s, x(\varphi(s))) d s, \quad t \in I .
$$

Assume that the following conditions are satisfied:

(i) $g \in C(I)$ is nonincreasing and nonnegative on the interval $I$;

(ii) $f: I \times R_{+} \rightarrow R_{+}$is continuous and there exists a nondecreasing function $m$ : $R_{+} \rightarrow R_{+}$such that the inequality

$$
|f(s, x)| \leq m(|x|)
$$

holds for all $s \in I$ and $x \in R$;

(iii) The operator $T: C(I) \rightarrow C(I)$ is continuous and satisfies the Darbo condition for the measure of noncompactness $\mu$ with a constant $a \geq 0$. Moreover, $T$ is a positive operator, i.e. $T x \geq 0$ if $x \geq 0$;

(iv) There exists a nonnegative constant $q$ such that

$$
|(T x)(t)| \leq q\|x\|
$$

for each $x \in C(I)$ and $t \in I$;

(v) $k: I \times I \rightarrow R_{+}$is integrable and nonincreasing in the first argument and

$$
K=\sup \left\{\int_{0}^{t}|k(t, s)| d s: t, s \in I\right\}
$$

(vi) $\varphi: I \rightarrow I$ is increasing and continuous function;

(vii) There exists $r_{o}>0$ with $\|g\|+K q m\left(r_{o}\right) r_{o}<r_{o}$ and $K m\left(r_{0}\right) a<1$.

Now, we are ready to state the existence theorem.

Theorem 2. Let the assumptions (i)-(vii) be satisfied, then equation (1.1) has at least one positive and nonincreasing solution $x \in C(I)$. 
Proof. Let us consider the operators $V, G$ defined on the space $C(I)$ in the following way:

$$
(V x)(t)=g(t)+(T x)(t) \int_{0}^{t} k(t, s) f(s, x(\varphi(s))) d s, \quad t \in[0, M]
$$

and

$$
(G x)(t)=\int_{0}^{t} k(t, s) f(s, x(\varphi(s))) d s .
$$

Firstly, we prove that if $x \in C(I)$ then $V x \in C(I)$. To do this it is sufficient to show that if $x \in C(I)$ then $G x \in C(I)$. Fix $\epsilon>0$, let $x \in C(I)$ and $t, s \in I$ such that $t \leq s$ and $|t-s| \leq \epsilon$. Then

$$
\begin{aligned}
&(G x)(s)-(G x)(t)=\int_{0}^{s} k(s, \tau) f(\tau, x(\varphi(\tau))) d \tau-\int_{0}^{t} k(t, \tau) f(\tau, x(\varphi(\tau))) d \tau \\
&=\int_{0}^{t} k(s, \tau) f(\tau, x(\varphi(\tau))) d \tau+\int_{t}^{s} k(s, \tau) f(\tau, x(\varphi(\tau))) d \tau \\
&-\int_{0}^{t} k(t, \tau) f(\tau, x(\varphi(\tau))) d \tau=\int_{0}^{t} k(s, \tau) f(\tau, x(\varphi(\tau))) d \tau \\
&-\int_{0}^{t} k(t, \tau) f(\tau, x(\varphi(\tau))) d \tau+\int_{t}^{s} k(s, \tau) f(\tau, x(\varphi(\tau))) d \tau \\
& \leq \int_{0}^{t} k(s, \tau) f(\tau, x(\varphi(\tau))) d \tau-\int_{0}^{t} k(s, \tau) f(\tau, x(\varphi(\tau))) d \tau \\
&+\int_{t}^{s} k(s, \tau) f(\tau, x(\varphi(\tau))) d \tau
\end{aligned}
$$

Now

$$
\begin{aligned}
|(G x)(s)-(G x)(t)| & \leq \int_{t}^{s}|k(s, \tau) f(\tau, x(\varphi(\tau)))| d \tau \\
& \leq \int_{t}^{s} k(s, \tau) m(|x(\varphi(\tau))|) d \tau \\
& \leq \int_{t}^{s} k(s, \tau) m(\|x\|) d \tau
\end{aligned}
$$

we obtain that

$$
|(G x)(s)-(G x)(t)| \leq m(\|x\|) \int_{t}^{s} k(s, \tau) d \tau
$$


Now, in virtue of the Lebesgue dominated Theorem we have that $\int_{t}^{s} k(s, \tau) d \tau \rightarrow 0$ as $\epsilon \rightarrow 0$. Thus $G x \in C(I)$ and consequentially, $V x \in C(I)$. Moreover, for each $t \in I$ we have

$$
\begin{aligned}
|(V x)(t)| & \leq|g(t)|+|(T x)(t)|\left|\int_{0}^{t} k(t, s) f(s, x(\varphi(s))) d s\right| \\
& \leq\|g\|+q\|x\| \int_{0}^{t} k(t, s) m(|x(\varphi(s))|) d s \\
& \leq\|g\|+q\|x\| m(\|x\|) \int_{0}^{t} k(t, s) d s \\
& \leq\|g\|+K q m(\|x\|)\|x\| .
\end{aligned}
$$

Hence,

$$
\|V x\| \leq\|g\|+K q m(\|x\|)\|x\| .
$$

Thus, if $\|x\| \leq r_{o}$ we obtain from assumption (vii) that

$$
\|V x\| \leq\|g\|+K q m\left(r_{o}\right) r_{o} \leq r_{o} .
$$

As a result the operator $V$ transforms the ball $B_{r_{0}}$ into itself.

In what follows, we will consider the operator $V$ on the subset $B_{r_{0}}^{+}$of the ball $B_{r_{0}}$ defined in the following way :

$$
B_{r_{0}}^{+}=\left\{x \in B_{r_{0}}: x(t) \geq 0, \text { for } t \in I\right\} .
$$

Obviously, the set $B_{r_{0}}^{+}$is nonempty, bounded, closed and convex. Let $x \in B_{r_{0}}^{+}$. Notice that in view of our assumptions (i)-(iv) if $x(t) \geq 0$ then $(V x)(t) \geq 0$ for $t \in I$. Thus $V$ transforms the set $B_{r_{0}}^{+}$into itself.

Now, we show that $V$ is continuous on the set $B_{r_{0}}^{+}$. To do this, let us fix $\epsilon>0$ and take arbitrary $x, y \in B_{r_{0}}^{+}$such that $\|x-y\| \leq \epsilon$. Then, for $t \in I$, we derive the following estimates:

$$
\begin{aligned}
\mid & (V x)(t)-(V y)(t) \mid \\
& =\left|(T x)(t) \int_{0}^{t} k(t, s) f(s, x(\varphi(s))) d s-(T y)(t) \int_{0}^{t} k(t, s) f(s, y(\varphi(s))) d s\right| \\
& \leq\left|(T x)(t) \int_{0}^{t} k(t, s) f(s, x(\varphi(s))) d s-(T y)(t) \int_{0}^{t} k(t, s) f(s, x(\varphi(s))) d s\right| \\
& +\left|(T y)(t) \int_{0}^{t} k(t, s) f(s, x(\varphi(s))) d s-(T y)(t) \int_{0}^{t} k(t, s) f(s, y(\varphi(s))) d s\right| \\
& \leq|(T x)(t)-(T y)(t)| \int_{0}^{t} k(t, s) f(s, x(\varphi(s))) d s \\
& +|(T y)(t)| \int_{0}^{t} k(t, s)|f(s, x(\varphi(s)))-f(s, y(\varphi(s)))| d s
\end{aligned}
$$




$$
\begin{aligned}
& \leq\|T x-T y\| \int_{0}^{t} k(t, s) m(|x(\varphi(s))|) d s \\
& +q\|y\| \int_{0}^{t} k(t, s) \beta_{r_{0}}(\epsilon) d s \\
& \leq\|T x-T y\| \int_{0}^{t} k(t, s) m(\|x\|) d s+q\|y\| \beta_{r_{0}}(\epsilon) \int_{0}^{t} k(t, s) d s \\
& \leq K m(\|x\|)\|T x-T y\|+K q\|y\| \beta_{r_{0}}(\epsilon), \\
& \leq K m\left(r_{o}\right)\|T x-T y\|+K q r_{0} \beta_{r_{0}}(\epsilon),
\end{aligned}
$$

where we denoted

$$
\beta_{r_{0}}(\epsilon)=\sup \left\{|f(u, x(u))-f(u, y(u))|: u \in I, x, y \in\left[0, r_{0}\right],\|x-y\| \leq \epsilon\right\} .
$$

Obviously, $\beta_{r_{0}}(\epsilon) \rightarrow 0$ as $\epsilon \rightarrow 0$ which is a simple consequence of the uniform continuity of the function $f$ on $I \times\left[0, r_{0}\right]$.

From the above estimate, we can write the following inequality:

$$
\|V x-V y\| \leq K m\left(r_{o}\right)\|T x-T y\|+K q r_{0} \beta_{r_{0}}(\epsilon)
$$

which implies the continuity of the operator $V$ on the set $B_{r_{0}}^{+}$.

In what follows, let us take a nonempty set $X \subset B_{r_{0}}^{+}$. Further, fix arbitrary a number $\epsilon>0$ and choose $x \in X$ and $t, s \in I$ such that $|t-s| \leq \epsilon$ and $t \leq s$. Then by our assumptions we have

$$
\begin{aligned}
& |(V x)(s)-(V x)(t)| \leq|g(s)-g(t)| \\
& +\left|(T x)(s) \int_{0}^{s} k(s, \tau) f(\tau, x(\varphi(\tau))) d \tau-(T x)(t) \int_{0}^{t} k(t, \tau) f(\tau, x(\varphi(\tau))) d \tau\right| \\
& \leq|g(s)-g(t)|+\mid(T x)(s) \int_{0}^{s} k(s, \tau) f(\tau, x(\varphi(\tau))) d \tau \\
& \quad-\quad(T x)(t) \int_{0}^{s} k(s, \tau) f(\tau, x(\varphi(\tau))) d \tau \mid \\
& +\left|(T x)(t) \int_{0}^{s} k(s, \tau) f(\tau, x(\varphi(\tau))) d \tau-(T x)(t) \int_{0}^{s} k(t, \tau) f(\tau, x(\varphi(\tau))) d \tau\right| \\
& +\left|(T x)(t) \int_{0}^{s} k(t, \tau) f(\tau, x(\varphi(\tau))) d \tau-(T x)(t) \int_{0}^{t} k(t, \tau) f(\tau, x(\varphi(\tau))) d \tau\right|
\end{aligned}
$$




$$
\begin{aligned}
& \leq \quad|g(s)-g(t)|+|(T x)(s)-(T x)(t)| \int_{0}^{s} k(s, \tau) m(|x(\varphi(\tau))|) d \tau \\
& +\quad|(T x)(t)| \int_{0}^{s}|k(s, \tau)-k(t, \tau)| m(|x(\varphi(\tau))|) d \tau \\
& +\quad|(T x)(t)| \int_{t}^{s} k(t, \tau) m(|x(\varphi(\tau))|) d \tau \\
& \leq \quad \omega(g, \epsilon)+m(\|x\|) \omega(T x, \epsilon) \int_{0}^{s} k(s, \tau) d \tau \\
& +q \quad\|x\| m(\|x\|) \int_{0}^{s}|k(s, \tau)-k(s, \tau)| d \tau \\
& +\quad q\|x\| m(\|x\|) \int_{t}^{s} k(t, \tau) d \tau \\
& \leq \omega(g, \epsilon)+K m(\|x\|) \omega(T x, \epsilon)+q\|x\| m(\|x\|) \int_{t}^{s} k(t, \tau) d \tau \\
& \leq \omega(g, \epsilon)+K m\left(r_{0}\right) \omega(T x, \epsilon)+q r_{o} m\left(r_{0}\right) \int_{t}^{s} k(t, \tau) d \tau .
\end{aligned}
$$

Hence

$$
\omega_{o}(V X) \leq K m\left(r_{o}\right) \omega_{o}(T X)
$$

Now, fix arbitrarily $x \in X$ and $t, s \in I$ such that $t \leq s$. Then we have the following chain of estimates:

$$
\begin{aligned}
\mid & (V x)(t)-(V x)(s) \mid-[(V x)(t)-(V x)(s)] \\
= & \mid g(t)+(T x)(t) \int_{0}^{t} k(t, \tau) f(\tau, x(\varphi(\tau))) d \tau \\
- & g(s)-(T x)(s) \int_{0}^{s} k(s, \tau) f(\tau, x(\varphi(\tau))) d \tau \mid \\
- & \mid g(t)+(T x)(t) \int_{0}^{t} k(t, \tau) f(\tau, x(\varphi(\tau))) d \tau-g(s) \\
- & \left.(T x)(s) \int_{0}^{s} k(s, \tau) f(\tau, x(\varphi(\tau))) d \tau\right] \leq\{|g(t)-g(s)|-[g(t)-g(s)]\} \\
+ & \left|(T x)(t) \int_{0}^{t} k(t, \tau) f(\tau, x(\varphi(\tau))) d \tau-(T x)(s) \int_{0}^{s} k(s, \tau) f(\tau, x(\varphi(\tau))) d \tau\right| \\
- & {\left[(T x)(t) \int_{0}^{t} k(t, \tau) f(\tau, x(\varphi(\tau))) d \tau-(T x)(s) \int_{0}^{s} k(s, \tau) f(\tau, x(\varphi(\tau))) d \tau\right] } \\
\leq & \left|(T x)(t) \int_{0}^{t} k(t, \tau) f(\tau, x(\varphi(\tau))) d \tau-(T x)(s) \int_{0}^{t} k(t, \tau) f(\tau, x(\varphi(\tau))) d \tau\right| \\
+ & \left|(T x)(s) \int_{0}^{t} k(t, \tau) f(\tau, x(\varphi(\tau))) d \tau-(T x)(s) \int_{0}^{t} k(s, \tau) f(\tau, x(\varphi(\tau))) d \tau\right|
\end{aligned}
$$




$$
\begin{aligned}
& +\left|(T x)(s) \int_{0}^{t} k(s, \tau) f(\tau, x(\varphi(\tau))) d \tau-(T x)(s) \int_{0}^{s} k(s, \tau) f(\tau, x(\varphi(\tau))) d \tau\right| \\
& -\left\{\left[(T x)(t) \int_{0}^{t} k(t, \tau) f(\tau, x(\varphi(\tau))) d \tau-(T x)(s) \int_{0}^{t} k(t, \tau) f(\tau, x(\varphi(\tau))) d \tau\right]\right. \\
& +\quad\left[(T x)(s) \int_{0}^{t} k(t, \tau) f(\tau, x(\varphi(\tau))) d \tau-(T x)(s) \int_{0}^{t} k(s, \tau) f(\tau, x(\varphi(\tau))) d \tau\right] \\
& \left.\left.+\mid(T x)(s) \int_{0}^{t} k(s, \tau) f(\tau, x(\varphi(\tau))) d \tau-(T x)(s) \int_{0}^{s} k(s, \tau) f(\tau, x(\varphi(\tau))) d \tau\right]\right\} \\
& \leq|(T x)(t)-(T x)(s)| \int_{0}^{t} k(t, \tau) f(\tau, x(\varphi(\tau))) d \tau \\
& +|(T x)(s)| \int_{0}^{t}|k(t, \tau)-k(s, \tau)| f(\tau, x(\varphi(\tau))) d \tau \\
& +|(T x)(s)| \int_{s}^{t} k(s, \tau) f(\tau, x(\varphi(\tau))) d \tau \\
& -\quad[(T x)(t)-(T x)(s)] \int_{0}^{t} k(t, \tau) f(\tau, x(\varphi(\tau))) d \tau \\
& -(T x)(s) \int_{0}^{t}(k(t, \tau)-k(s, \tau)) f(\tau, x(\varphi(\tau))) d \tau \\
& -(T x)(s) \int_{s}^{t} k(s, \tau) f(\tau, x(\varphi(\tau))) d \tau .
\end{aligned}
$$

Since $f \geq 0, k \geq 0$ and $t \rightarrow k(t, s)$ is nonincreasing then we have

$$
\int_{0}^{t}(k(t, \tau)-k(s, \tau)) f(\tau, x(\varphi(\tau))) d \tau \geq 0
$$

and

$$
\int_{s}^{t} k(s, \tau) f(\tau, x(\varphi(\tau))) d \tau \rightarrow 0 \text { as } \epsilon \rightarrow 0 .
$$

Finally, (2.2)-(2.3) imply

$$
\begin{aligned}
& |(V x)(t)-(V x)(s)|-[(V x)(t)-(V x)(s)] \\
\leq & \{|(T x)(t)-(T x)(s)|-[(T x)(t)-(T x)(s)]\} \int_{0}^{t} k(t, \tau) f(\tau, x(\varphi(\tau))) d \tau \\
\leq & \{|(T x)(t)-(T x)(s)|-[(T x)(t)-(T x)(s)]\} \int_{0}^{t} k(t, \tau) m(|x(\varphi(\tau))|) d \tau \\
\leq & \{|(T x)(t)-(T x)(s)|-[(T x)(t)-(T x)(s)]\} \int_{0}^{t} k(t, \tau) m(\|x\|) d \tau \\
\leq & m\left(r_{0}\right)\{|(T x)(t)-(T x)(s)|-[(T x)(t)-(T x)(s)]\} \int_{0}^{t} k(t, \tau) d \tau \\
= & K m\left(r_{0}\right) i(T x) .
\end{aligned}
$$


Hence, we get

$$
i(V x) \leq K m\left(r_{0}\right) i(T x)
$$

and consequently,

$$
i(V X) \leq K m\left(r_{0}\right) i(T X) .
$$

Finally, by the equations (2.1)-(2.4) we obtain

$$
\mu(V X) \leq K m\left(r_{0}\right) \mu(T X) \leq K m\left(r_{0}\right) a \mu(X) .
$$

Now, since $K m\left(r_{0}\right) a<1$ and applying Theorem 1, we complete the proof.

Remark 4. By Remarks 1 and 2, we have that solutions of the integral equation (1.1) belonging to the set $B_{r_{0}}^{+}$are positive, nonincreasing and continuous on the interval $I=[0, M]$.

Now, we provide an example illustrating the applicability of Theorem 2.

For example, taking in the assumption (iv) $q=1$ and putting $T x=x$ for $x \in C(I)$ we obtain the Volterra integral equation of the form

$$
x(t)=g(t)+x(t) \int_{0}^{t} k(t, s) f(s, x(\varphi(s))) d s, \quad t \in I .
$$

Obviously that equation is a particular of equation (1.1).

\subsection{Convolution type}

Consider the quadratic integral equation of convolution type of the form

$$
x(t)=g(t)+(T x)(t) \int_{0}^{t} k(t-s) f(s, x(\varphi(s))) d s, t \in[0, M] .
$$
(2.5) .

Now, the following Corollary deals with the integral equation of convolution type

Corollary 1. Let $k: I \rightarrow R_{+}$be nonincreasing function and let the assumptions of Theorem 2 be satisfied, then equation (2.5) has at least one positive and nonincreasing solution $x \in C(I)$.

\subsection{Fractional order equation}

Now, taking $k(t-s)=\frac{(t-s)^{\alpha-1}}{\Gamma(\alpha)}$, then we have the following Corollary.

Corollary 2. Let $M^{\alpha} m\left(r_{o}\right) a<\Gamma(\alpha+1)$. Then under the assumptions (i)-(iv) and (vi) of Theorem 2, the nonlinear quadratic functional integral equation of fractional order

$$
x(t)=g(t)+(T x)(t) \int_{0}^{t} \frac{(t-s)^{\alpha-1}}{\Gamma(\alpha)} f(s, x(\varphi(s))) d s, t \in[0, M], 0<\alpha<1 .
$$

has at least one positive and nonincreasing solution $x \in C(I)$. 
Corollary 3. Let $(T x)(t)=p(t, x(\psi(t)))$ and $I=[0,1]$ in Corollary 2, we obtain the same result as was proved in [17].

Corollary 4. Under the same assumptions (i), (ii) and (vi) of Theorem 2 (with $q=1$ and $(T x)(t)=x(t))$, then the fractional-order integral equation

$$
x(t)=g(t)+x(t) \int_{0}^{t} \frac{(t-s)^{\alpha-1}}{\Gamma(\alpha)} f(s, x(\varphi(s))) d s, t \in[0, M], 0<\alpha<1 .
$$

has at least one positive and nonincreasing solution $x \in C(I)$ if $M^{\alpha} m\left(r_{o}\right)<\Gamma(\alpha+1)$.

\section{References}

[1] R.P. Agarwal, D. O'Regan, Global existence for nonlinear operator inclusion, Comput. Math. Appl. 38 (11-12) (1999) 131-139.

[2] R.P. Agarwal, D. O'Regan, P.J.Y. Wong, Positive Solutions of Differential, Difference and Integral Equations, Kluwer Academic, Dordecht, 1999.

[3] J. Banaś, Measures of noncompactness in the space of continuous tempered functions, Demonstratio Math. 14 (1981) 127-133.

[4] J. Banaś, K. Goebel, Measures of Noncompactness in Banach Spaces, Lecture Notes in Pure and Applied Mathematics, Vol. 60, Marcel Dekker, New York, 1980.

[5] J. Banaś, L. Olszowy, Measures of noncompactness related to monotonicity, Comment. Math. 41 (2001) 13-23.

[6] J. Banaś, A. Martinon, Monotonic solutions of a quadratic integral equation of Volterra type, Comput. Math. Appl. 47 (2004) 271-279.

[7] J. Banaś, M. Lecko, W.G. El-Sayed, Existence theorems for some quadratic integral equations, J. Math. Anal. Appl. 222 (1998) 276-285.

[8] J. Banaś, K. Sadarangani, Solvability of Volterra-Stieltjes operator-integral equations and their applications, Comput. Math. Appl 41 (2001) 1535-1544.

[9] J. Banaś, J.R. Rodriguez, K. Sadarangani, On a class of Urysohn-Stieltjes quadratic integral equations and their applications, J. Comput. Appl. Math. 113 (2000) 35-50.

[10] J. Banaś, J. Rocha, K.B. Sadarangani, Solvability of a nonlinear integral equation of Volterra type, J. Comput. Appl. Math. 157 (2003) 31-48.

[11] J. Banaś, B. Rzepka, On existence and asymptotic stability of solutions of a nonlinear integral equation, J. Math. Anal. Appl. 284 (2003) 165-173. 
[12] J. Banaś, J.R. Rodriguez, K. Sadarangani, On a nonlinear quadratic integral equation of Urysohn-Stieltjes type and its applications, Nonlinear Anal. 47 (2001) $1175-1186$.

[13] J. Banaś, B. Rzepka, An applications of a measure of noncompactness in the study of asymptotic stability, Appl. Math. Lett. 16 (2003) 1-6.

[14] B. Cahlon, M. Eskin, Existence theorems for an integral equation of the Chandrasekhar H-equation with perturbation, J. Math. Anal. Appl. 83 (1981) 159-171.

[15] C. Corduneanu, Integral Equations and Applications, Cambridge University Press, Cambridge, MA, 1991.

[16] G. Darbo, Punti uniti in trasformazioni a codominio non compatto, Rend. Sem. Mat. Univ. Padova 24 (1955) 84-92.

[17] A.M.A. EL-Sayed, H.H.G. Hashem, Existence results for nonlinear quadratic functional integral equations of fractional orders, Miskolc Mathematical Notes 1 (2013) 79-88.

[18] A.M.A. EL-Sayed, H.H.G. Hashem, Integrable and continuous solutions of a nonlinear quadratic integral equation, Electron. J. Qual. Theory Differ. Equ. 25 (2008) $1-10$.

[19] A.M.A. EL-Sayed, H.H.G. Hashem, Monotonic solutions of functional integral and differential equations of fractional order, Electron. J. Qual. Theory Differ. Equ. (2009) 1-8.

[20] A.M.A. EL-Sayed, H.H.G. Hashem, Monotonic positive solution of a nonlinear quadratic functional integral equation, Appl. Math. Comput. vol. 216 (2010) $2576-2580$.

[21] W.G. EL-Sayed, B. Rzepka, Nondecreasing solutions of a quadratic integral equation of Urysohn type, Comput. Math. Appl. 51 (2006) 1065-1074.

[22] S. Hu, M. Khavani, W. Zhuang, Integral equations arising in the kinetic theory of gases, Appl. Anal. 34 (1989) 261-266.

[23] D. O'Regan, M. Meehan, Existence Theory for Nonlinear Integral and Integrodifferential Equations, Kluwer Academic, Dordrecht, 1998. 
Nonincreasing Solutions for Quadratic Integral Equations of Convolution Type 107

DOI: $10.7862 / \mathrm{rf} .2019 .6$

W.G. El-Sayed

email: wagdygoma@alexu.edu.eg

ORCID: 0000-0003-1271-2681

Faculty of Science

Alexandria University

Alexandria

EGYPT

A.A.H. Abd El-Mowla

email: aziza.abdelmwla@yahoo.com

ORCID: 0000-0002-7160-4448

Faculty of Science

Omar Al-Mukhtar University

Derna

LIBYA

Received 21.02.2019

Accepted 15.06.2019 